\title{
A Substrate for Small Patch Antennas Providing Tunable Miniaturization Factors
}

\author{
Kevin Buell, Student Member, IEEE, Hossein Mosallaei, Senior Member, IEEE, and Kamal Sarabandi, Fellow, IEEE
}

\begin{abstract}
Magnetic properties were imparted to a naturally nonmagnetic material by metallic inclusions. A patch antenna tested the performance of the magnetic metamaterial as a substrate and validated that a single substrate can achieve a range of miniaturization values. The effective medium metamaterial substrate employed electromagnetically small embedded circuits (ECs) to achieve permeability and permittivity greater than that of the host dielectric. Geometric control of the ECs allowed $\mu$ and $\epsilon$ to be tailored to the application. The magnetic metamaterial exhibited enhanced $\mu$ and $\epsilon$ with acceptable loss-factor levels. Models for predicting $\mu$ and $\epsilon$ are presented, the benefits of employing metamaterial substrates are discussed, and the results in this antenna experiment are presented. The metamaterial exhibits performance characteristics not achievable from natural materials. Of particular significance is that with the permeability varying strongly and predictably with frequency, the miniaturization factor may be selected by tuning the operating frequency. Simulations indicate that such performance can be extended to several gigahertz with current technology. Relative permeability values in the $\mu_{r}=1-5$ range are achievable for moderately low-loss applications. Representative antenna miniaturization factors on the order of 4-7 over a moderate (approximately $10 \%$ ) transmission bandwidth and efficiencies in a moderate range $(20 \%-35 \%)$ are demonstrated with the possibility of higher efficiencies indicated.
\end{abstract}

Index Terms-Magnetic materials, metamaterials, microstrip antennas.

\section{INTRODUCTION}

\section{A. Background and Goals}

$\mathbf{T}$ HE GOAL of this study is to develop and experimentally validate engineered magnetic materials with properties that do not exist in natural materials. We experimentally demonstrate a technique of producing magnetic properties in an engineered material using only nonmagnetic elements [1]-[4]. The application chosen to demonstrate the magnetic permeability of this engineered material, which will be referred to as a metamaterial, is that of a miniaturized patch antenna above

Manuscript received October 19, 2004; revised May 24, 2005 and August 23, 2005.

K. Buell is with the Department of Electrical Engineering and Computer Science, The University of Michigan at Ann Arbor, Ann Arbor, MI 48109-2122 USA (e-mail: kbuell@umich.edu).

H. Mosallaei was with the Department of Electrical Engineering and Computer Science, The University of Michigan at Ann Arbor, Ann Arbor, MI 48109-2122 USA. He is now with the College of Engineering, Northeastern University, Boston, MA 02115 USA.

K. Sarabandi is with the Radiation Laboratory, The University of Michigan at Ann Arbor, Ann Arbor, MI 48109-2122 USA (e-mail: saraband@eecs.umich.edu).

Digital Object Identifier 10.1109/TMTT.2005.860329

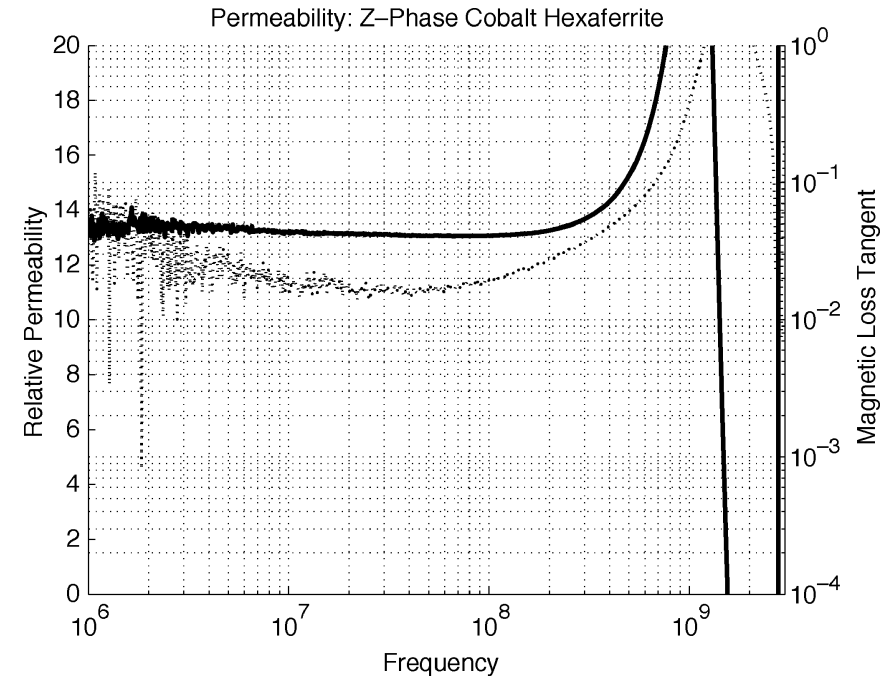

Fig. 1. Measured magnetic permeability and loss tangent of $Z$-phase Cobalt HexaFerrite (solid and dashed lines, respectively).

a ground plane. While there are numerous alternative methods for miniaturizing patch antennas, and a few alternative methods of producing magnetic properties using embedded circuits (ECs) [5], [6] the purpose of this study is to experimentally validate our EC metamaterial models and show that for a single substrate, the miniaturization factor can be tuned by selection of the operating frequency. The patch antenna application calls for low-loss operation with a specifiable relative magnetic permeability at frequencies where low-loss magnetically permeable materials do not already exist.

For low-loss applications in the microwave region, natural material choices are limited to nonmagnetic dielectrics. Unfortunately, for magnetic materials, the upper frequency end of the magnetic region for high-quality ferrites, limited by the gyromagnetic resonances, occurs in the VHF-UHF range, which is too low a frequency for microwave applications.

Fig. 1 shows the relative permeability and magnetic loss tangent of $Z$-phase Cobalt HexaFerrite developed by the TransTech Corporation, Adamstown, MD. This $Z$-phase Cobalt HexaFerrite is representative of the current upper frequency limit for low-loss magnetic permeability from natural materials. The admittedly subjective maximum "useful" frequency for this material is approximately $500 \mathrm{MHz}$. To our knowledge, there is no material currently available with moderately low loss $\left(\tan \delta_{m}=\left(\mu^{\prime \prime} / \mu^{\prime}\right)<0.02\right)$ and moderately enhanced permeability $\left(\mu_{r}^{\prime}>2\right)$ for operation in the microwave region. An engineered material such as our metamaterial, which can fit this need, would be quite useful. 


\section{B. Benefits of Magnetic Materials}

The permittivity of a composite dielectric can be selectively engineered by the mixing of low and high dielectric materials to provide low-loss and high performance throughout the microwave operating region for practically any desirable permittivity. For example, alumina $\left(\mathrm{Al}_{2} \mathrm{O}_{3}\right)$ has a dielectric constant of approximately $\epsilon_{r}=10\left(\tan \delta_{e} \leq 3 \times 10^{-4}\right.$ at $\left.10 \mathrm{GHz}\right)$ and can be mixed in controlled ratios with lower dielectric buffer materials to achieve any desirable dielectric constant from $\epsilon_{r}=2-10$ while maintaining an acceptable low-loss factor. Similar, but more challenging to process is titania $\left(\mathrm{TiO}_{2}\right)$, which has a very low dielectric-loss tangent $\left(\tan \delta_{e} \leq 1 \times 10^{-3}\right.$ at $\left.10 \mathrm{GHz}\right)$ and a dielectric constant of close to $\epsilon_{r}=100$, which opens up the entire possible range of dielectric values [7]. In contrast to the wide variety of low-loss dielectrics available, the permeability of low-loss natural materials and their various composites are effectively limited to that of free space in the microwave region.

Currently for microwave applications, dielectric materials are chosen to achieve the desired electromagnetic (EM) phenomenon of the application goals. High dielectric-constant materials are used to achieve EM scaling, field confinement, and other useful benefits. Limiting extremely high dielectrics from many desirable applications is the dramatic mismatch in wave impedance for the material relative to feed networks and free space.

If permeability can be increased from that of free space, the product of $\mu$ and $\epsilon$ increases quickly with relative permeability, providing miniaturization and EM scaling $\left(\lambda_{\text {medium }}=\left(\lambda_{0} / \sqrt{\mu_{r} \epsilon_{r}}\right)\right)$. In the geometry of the patch antenna, the majority energy storage mechanism is capacitive in the electric field. By increasing the amount of magnetic energy storage, the magnetic-electric imbalance is reduced and the system bandwidth automatically improves [3]. Since the patch antenna is so strongly capacitive, a $\mu_{r}>\epsilon_{r}$ condition would be preferred in terms of balancing energy storage mechanisms. In terms of minimizing the free energy trapped within the substrate, a $\mu_{r}=\epsilon_{r}$ condition would be preferred. As relative permeability increases to match relative permittivity, the intrinsic impedance of the medium $\left(\eta_{\text {medium }}=\sqrt{\mu_{r} \mu_{0} / \epsilon_{r} \epsilon_{0}}\right)$ approaches that of free space $\left(\eta_{0}=\sqrt{\mu_{0} / \epsilon_{0}}\right)$. It is the difference in intrinsic impedances $\eta_{0}$ and $\eta_{\text {medium }}$ that determines the reflection coefficients at the interface, and we can benefit from being able to control this factor independently of $\lambda_{\text {medium. }}$.

The benefit to minimizing the energy loss due to the reflection at this interface is obvious and further anticipated benefits are improvements in matching and bandwidth as a result of increasing the proportion of magnetic energy storage. Benefits in improved input matching for miniaturized devices and elimination of trapped surface waves can offer significant potential benefits for microstrip antennas if losses are minimal.

As we will show, the metamaterial presented provides a permeability that varies from $\mu_{r}=1-5$ over a reasonable operating band. This property allows the designer to make an efficiency/miniaturization tradeoff with a single substrate material. For a specified efficiency level, the maximum miniaturization factor may be selected, thereby enabling various antennas to op- erate on the same physical metamaterial substrate while each experiences a different miniaturization factor and efficiency.

1) Natural Magnetic Materials: The bulk permeability of a material is a quantitative description of how readily the material experiences magnetization, wherein the materials charges align their movement with an externally applied magnetic field.

A circulating charge produces a magnetic moment, and the magnetic moments of atoms are the building blocks of natural magnetics. The macroscopic magnetic properties of materials are the consequence of magnetic moments associated with individual electrons. The classes of observed magnets include diamagnetism, paramagnetism, and ferromagnetism. In addition, antiferromagnetism and ferrimagnetism are considered to be subclasses of ferromagnetism. Diamagnetism and paramagnetism exhibit very weak magnetic properties and are not adequate for normal EM applications. Ferromagnetism is exhibited in some metallic materials such as iron, cobalt, or nickel when magnetic moments due to uncanceled electron spins of adjacent atoms interact to align with one another and produce magnetic susceptibilities as high as $10^{6}$. This strong magnetization is of limited use for magneto-dielectrics due to the high conductivity and loss of the metals necessary to produce it. Antiferromagnetism occurs when the coupling interaction between adjacent atoms result in antiparallel alignment. These magnetic moments cancel one another, and there is no net magnetic moment.

Finally, and most promisingly is ferrimagnetism-a magnetism exhibited by some ceramics as a result of their complex crystal structure. In such ceramics, there are parallel and antiparallel coupling interactions between the ions, similar to in antiferromagnetism; however, the net ferrimagnetic moment arises from the incomplete cancellation of spin magnetic moments. [8]

In the presence of a magnetic field, the magnetic moments of a ferrimagnetic material tend to become aligned with the applied field and to reinforce it by virtue of their own magnetic fields. Fig. 1 shows the complex magnetic permeability $\left(\mu=\mu^{\prime}-\right.$ $\left.j \mu^{\prime \prime}\right)$ of a $Z$-phase Cobalt HexaFerrite ceramic as a function of frequency.

The challenge to microwave applications arises from the inertia of atomic systems. Although the mass of an electron is small, it is not zero, and the attempts of the electrons dipole moments to track an externally applied magnetic field deteriorate and eventually fail altogether for inertial reasons as the excitation field approaches and passes materials gyromagnetic resonance frequency. As gyromagnetic resonance is approached, the materials loss factor increases dramatically, as exhibited in Fig. 1, and above resonance, the material becomes essentially nonmagnetic. Unfortunately for microwave engineers, it appears that magneto-dielectrics produced from natural materials exhibit gyromagnetic resonance in the VHF-UHF region and are unusable for low-loss microwave applications.

If a mechanism similar to natural magnetics can be developed for microwave operation by synthetic means, low-loss operation may be pushed into the microwave region and low-loss microwave magneto-dielectrics may become a reality. Already various researchers have proven that it is possible to replicate magnetic behavior by inserting electromagnetically small metallic inclusions into a natural dielectric. [1]-[4], [6]. 


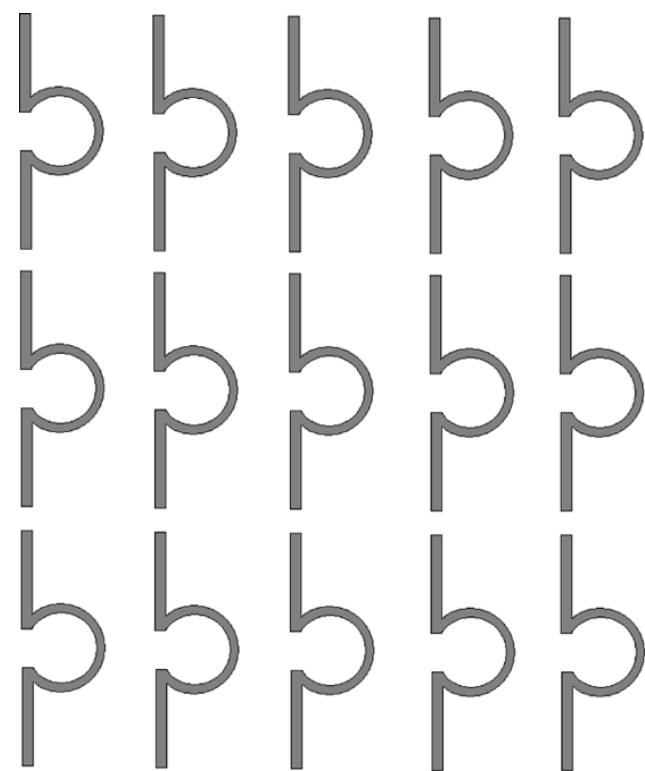

Fig. 2. Medium with metallic inclusions in the shape of the greek letter $\Omega$. The "omega" medium exhibits enhancement of both the magnetic and dielectric properties over the host material in certain frequency regions determined by the circuit resonances.

This paper shows the development and experimental analysis of a magneto-dielectric metamaterial in actual application for a miniaturized patch antenna whose miniaturization factor is a function of frequency.

\section{EC Metamaterials}

In 1968, Veselago [9] theoretically investigated the physics of materials with negative permeabilities and permittivities. When either permeability or permittivity is negative, EM waves cannot propagate in the medium. This nonpropagating phenomenon has been dubbed "bandgap" operation. Interestingly, as shown by Veselago, when both permeability and permittivity are negative, EM wave propagation is possible. Today such materials are most often referred to as "left-handed" materials due to the left-handed Poynting vector triplet that their electric, magnetic, and propagation vectors form.

Recent experimental work builds upon the theoretical development for left-handed materials provided by Veselago and is perhaps the most popular area of research for EC metamaterials [10]-[15].

In contrast to research on negative permeability and permittivity materials, the purpose of this paper is to achieve enhanced positive magnetic permeability and electric permittivity for lowloss microwave applications.

The concepts of using ECs to enhance dielectric properties or achieve magnetic properties in an otherwise nonmagnetic medium are not new, but we believe have not received adequate attention when the significance of the potential benefits are considered. In one relevant example, Saadoun and Engheta investigated a theoretical material they called the "omega" medium in the mid 1990s [5]. Their "omega" medium is composed of a host material with small inclusions shaped like the Greek letter $\Omega$ (see Fig. 2). Their theoretical analysis of EM wave interaction

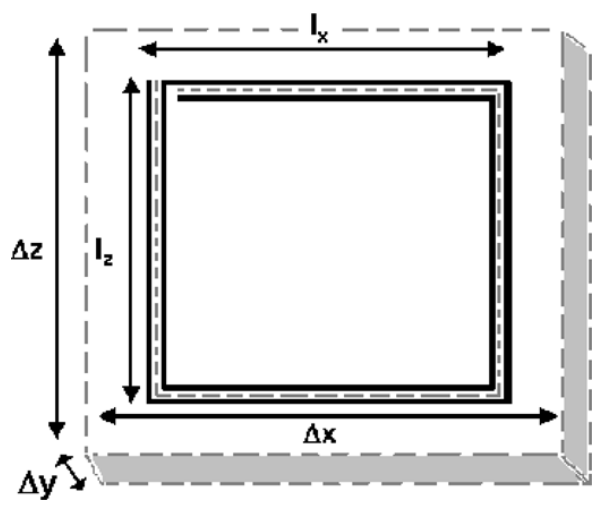

Fig. 3. Metamaterial unit cell. $\Delta_{x}, \Delta_{y}$, and $\Delta_{z}$ is the unit cell size. In this diagram, $N=2$ is the number of wraps of the spiral. To achieve permeability enhancement, the magnetic field shall be aligned along the $Y$-axis (normal to the page) and the electric field shall align along either the $X$ - or $Z$-axis.

with the circuit model for such a medium showed both an effective permittivity and an effective permeability, thus establishing both dielectric and magnetic enhancement.

Recently, geometries optimized to provide superior magnetic properties have been considered theoretically [1], [6], but to our knowledge, none have been proven useful in practical experimental application.

Our circuit geometry is engineered to control energy coupling and storage. The benefit of this control is that, within the limits of the processing technology, the effective permittivity and, most importantly, effective permeability of the medium, can be tailored to the demands of the application.

\section{EFFECTIVE MEDIUM OpERATION}

The storage of energy in magnetic fields is the definitive characteristic of a magnetically permeable material. When magnetic energy storage is achieved by means other than atomic electron orbital or spin phenomenon, an engineered effective bulk permeability is observed. The basic circuit unit for magnetic energy storage is the inductor, and an electromagnetically small inductor embedded into a dielectric material will store coupled magnetic energy in a manner similar to the means by which magnetic energy is stored in the electron orbital or spin motion of materials exhibiting natural magnetic permeability. This EC magnetic energy storage imparts an effective bulk permeability to the material.

Fig. 3 shows a single element of an EC capable of producing magnetic properties in a natural dielectric. The spiral loop acts as an inductor, coupling energy from an incident magnetic field to produce a current loop in the spiral. There is a distributed capacitance between the loops of the spiral, and the interaction between the spiral inductance and spiral capacitance causes the resonant behavior. Near resonance, the current magnitude in the spiral loop increases and the magnetic permeability is enhanced.

To achieve an effective medium behavior, the ECs must be distributed uniformly through the host dielectric. Planar microstrip processing is employed to form a two-dimensional array of the resonant spirals (see Fig. 4) and the resulting substrate-metallization layers are stacked to form a three-dimensional (3-D) effective medium. This method of assembly allows for the critical control of geometrically determined 


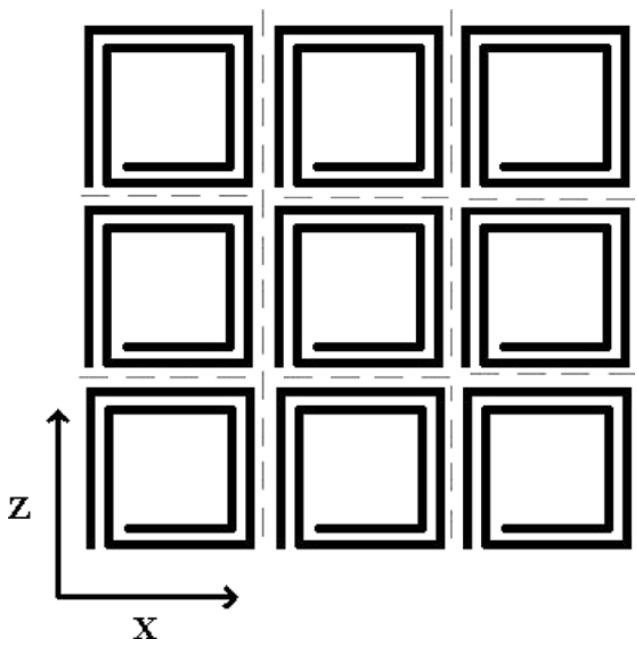

Fig. 4. Infinite metamaterial medium. A passive 2-D $X Z$ array of elements shown in Fig. 3. These circuit boards can be stacked in the $Y$-dimension to approximate an infinite magnetic medium.

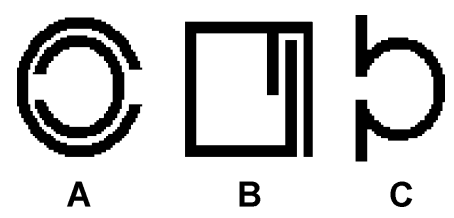

Fig. 5. Previous magnetic ECs. (a) Split-ring resonator. (b) Square $L C$ resonator. (c) "Omega" medium resonator.

circuit parameters and thereby selection of resonant frequency and coupling factors.

\section{A. Equivalent-Circuit Model}

Our effective medium employs passive ECs embedded in a dielectric medium for which we have developed the theoretical analytical models predicting material performance [1]-[4]. Previous researchers have developed means of achieving magnetism from passive ECs conductors [5], [6] and this study builds upon the existing state-of-the-art. One of our previous designs consisted of a single square spiral with an interdigitated capacitor providing lumped-element capacitance-like performance and is shown in Fig. 5 beside other magnetic EC resonators [4].

One drawback of this square $L C$ resonator is its nonoptimal use of unit cell area. A good design for optimal magnetic permeability would "enclose" as much of the unit-cell area as possible to achieve the highest coupling of incident magnetic energy while maximizing packing density. A square inductive loop seems to be a reasonable candidate and was the choice of our previous design. An interdigitated capacitor seemed like a reasonable choice to provide the capacitance with which the inductive loop would resonate, but we have since concluded that this is a nonoptimal use of the unit-cell area inasmuch as that the interdigitation consumes too much valuable space inside the inductive loop and thereby inhibits optimal coupling to the incident magnetic field. We have since concluded that a spiral loop is preferable inasmuch as it uses less area to provide equivalent

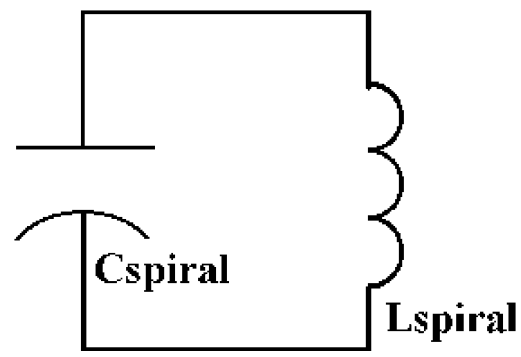

Fig. 6. Spiral-loop equivalent lumped-element circuit model.

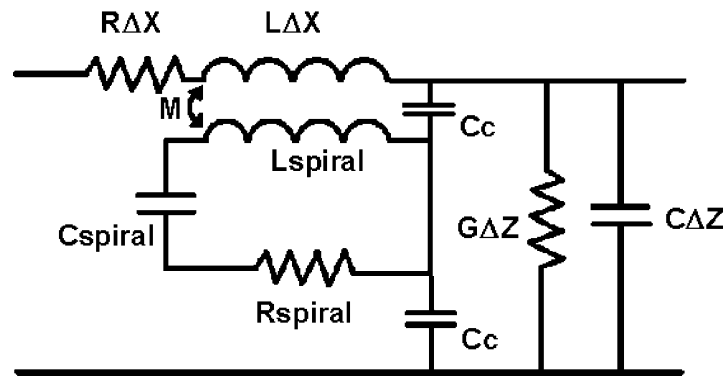

Fig. 7. Transmission-line equivalent model for magnetic metamaterial.

capacitance while simultaneously providing additional inductance and, hence, additional permeability.

These ECs (Fig. 3) couple incident magnetic energy to their inductive elements and store the energy in an $L C$ resonator.

The basic design equations for the spiral loop circuit would be helpful in understanding the operation of the metamaterial. Useful design equations would need to provide approximate lumped-element values for the distributed capacitance and inductance of a flat spiral inductor.

Although a theoretically rigorous analysis of the EC metamaterial is not available, a simple preliminary model is available, which provides an intuitive understanding of the EC metamaterial behavior. For quantitatively precision, numerical simulation is best employed as the second step in metamaterial design.

Toward the end of maximum physical understanding with only moderate analytic complexity, the spiral loop of Fig. 3 may be most simply modeled as an $L C$ resonator, as shown in Fig. 6. This simple resonator interacts with its host medium in a manner similar to the well studies behavior of a plasma near its resonance and, hence, the composite transmission-line equivalent model for the resonator is very similar to that for a plasma. Incorporating the spiral loop loss mechanisms $\left(R_{\text {spiral }}\right)$, the equivalent-circuit model for the composite medium is given in Fig. 7. Fig. 7 can best be interpreted as a classic $R L G C$ transmission-line model for a medium with an embedded $L C$ resonator inductively and capacitively coupled to it.

For the derivation of the circuit models and (1)-(12), the interested reader is directed to [2]. What follows here is a significantly expanded explanation and discussion of the design and validation process employing these methods for a more practical planar EC geometry.

1) Capacitance: To model the equivalent lumped-element capacitance $\left(C_{\text {spiral }}\right.$ in Fig. 6) of the spiral loop (Fig. 3), the primary capacitive effect to be considered is the capacitance between adjacent wraps of the spiral inductor. 


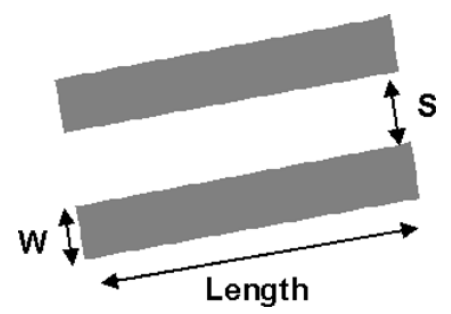

Fig. 8. Geometry for (3) to calculate capacitance of two flat coplanar metallic strips.

Additional smaller capacitances will result from the interaction between nonadjacent wraps, but only considering the adjacent wraps should give a preliminary understanding of the physics involved. These additional capacitances will be especially significant in cases where loops have greater than two wraps of the spiral arm. In this case, the nearest capacitive effect not included in the model is only twice the distance of the included elements.

The distributed capacitance of the spiral inductor embedded in the host dielectric can be determined by considering the geometry of the "capacitive spiral" indicated in Fig. 3 by a dotted line, which traces the path between the metallic spiral arms. The value of capacitance can be computed from (1) where $L_{\mathrm{SG}}$ is the length of the spiral gap given by (2) and the gap fraction $g$ is given by (3) as follows:

$$
\begin{aligned}
C_{\text {spiral }}= & \epsilon_{\text {diel }} \frac{K\left(\sqrt{1-g^{2}}\right)}{K(g)} L_{\mathrm{SG}} \\
L_{\mathrm{SG}}= & 2 n(l z+l x) \\
& -w\left[(2 n+2) \frac{2 n+1}{2}-1+(2 n+1) n\right] \\
& -s\left[(2 n+1) n+(2 n)\left(\frac{2 n-1}{2}+\frac{1}{2}\right)\right] \\
g= & \frac{\frac{s}{s}}{s^{2}+w} \\
K(g)= & \int_{0}^{\frac{\pi}{2}} \frac{d \phi}{\sqrt{1-g^{2} \sin ^{2} \phi}} .
\end{aligned}
$$

The basic form of the capacitive (1) is the capacitance per unit length of coplanar thin metallic strips multiplied by the length of the strips and the dielectric constant of the host medium. An elliptical integral (4) exists for determining the capacitance per unit length. In (1)-(3), the metallization thickness is assumed to be zero, $\epsilon_{\text {diel }}=\epsilon_{r \operatorname{diel}} \epsilon_{0}$ where $\epsilon_{0}$ is the permittivity of free space, the width of the trace metallization is indicated by $w$, and the inter-trace gap spacing is denoted by $s$ (see Fig. 8).

Equation (2) is an analytic formula for the length of the spiral gap. If $N$ is the number of turns of the metallic spiral arm, then $n=N-1$ is the number of turns of the capacitive spiral gap. This formula is correct for integer or half-integer values of $N$, and integer/ 4 values if $l x=l z$. For other values of $N$, the analytic formula provides a reasonable estimate to first order.

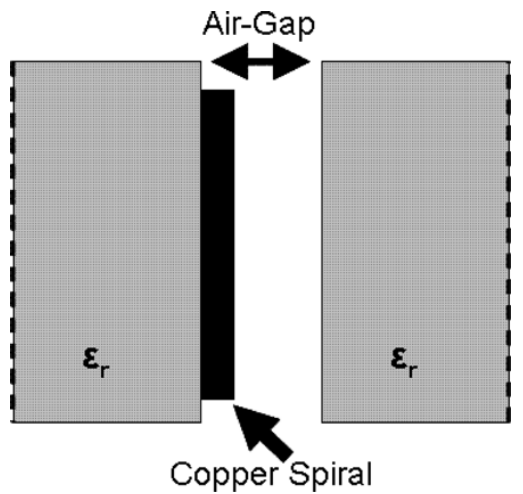

Fig. 9. Air gap caused by substrate warpage decreases the effective capacitance of the spiral resonator, increasing the metamaterial resonance frequency.

For our 250-MHz design, $l x=l z=16 \mathrm{~mm}, w=s=$ $0.127 \mathrm{~mm}$, and $L_{\mathrm{SG}}=6.24 \mathrm{~mm}$. For a Rogers RO-4003 dielectric host medium, $\epsilon_{r \text { diel }}=3.38$ and $C_{\text {spiral }}=5.3 \mathrm{pF}$.

Although this estimate of capacitance is acceptable, it does neglect additional capacitances between spiral elements in adjacent different metamaterial unit cells and corner and gap-end effects, as well as capacitance between nonadjacent wraps, nor does it account for the air gap between stacked layers (see Fig. 9). For these reasons, the capacitance predicted by (1) will be at best a rough approximation of the actual capacitance. Nevertheless, (1) provides useful insights into the behavior of the EC and is a useful starting point for design, and thus, is worthy of consideration.

2) Inductance: The planar elements in Fig. 4 are stacked along the $Y$-dimension with a spacing of $\Delta y$. This geometry effectively forms a solenoid along the $Y$-axis of spiral loop elements and, due to the long-solenoid structure, a uniform field distribution can be assumed. This observation provides the starting-point for modeling the spiral inductance. With this estimate in mind, for low values of spiral turns $N$, the inductance of a single spiral loop can be derived from (5) where $S$ is the cross-sectional area of the spiral as follows:

$$
L_{\text {spiral }}=\mu_{0} \frac{N^{2}}{\Delta y} S
$$

For our geometry, $\Delta y=3.028 \mathrm{~mm}, N=2$, and $S \approx$ $2.56 \times 10^{-4} \mathrm{~m}^{2}$. The inductance of a single spiral loop element provided by (5) is just slightly higher than the real value due to imperfect "fill-ratio." Basically, (5) assumes perfect magnetic linkage between all concentric loops of the spiral. Choosing an average area for the spiral (such as the area enclosed by the dashed line in Fig. 3) should account for the discrepancy. As in the capacitive calculation, the simplifying approximations of this calculation limits its accuracy. In addition to the imperfect magnetic linkage mentioned, a significant deviation from ideality occurs in that the current on each spiral element is forced to go to zero at its ends, a condition that does not exist in ideal solenoidal wrapped wires represented by (5). Nevertheless, it is illustrative to consider the inductance for our geometry, which is $L_{\text {spiral }}=0.425 \mu \mathrm{H}$. 


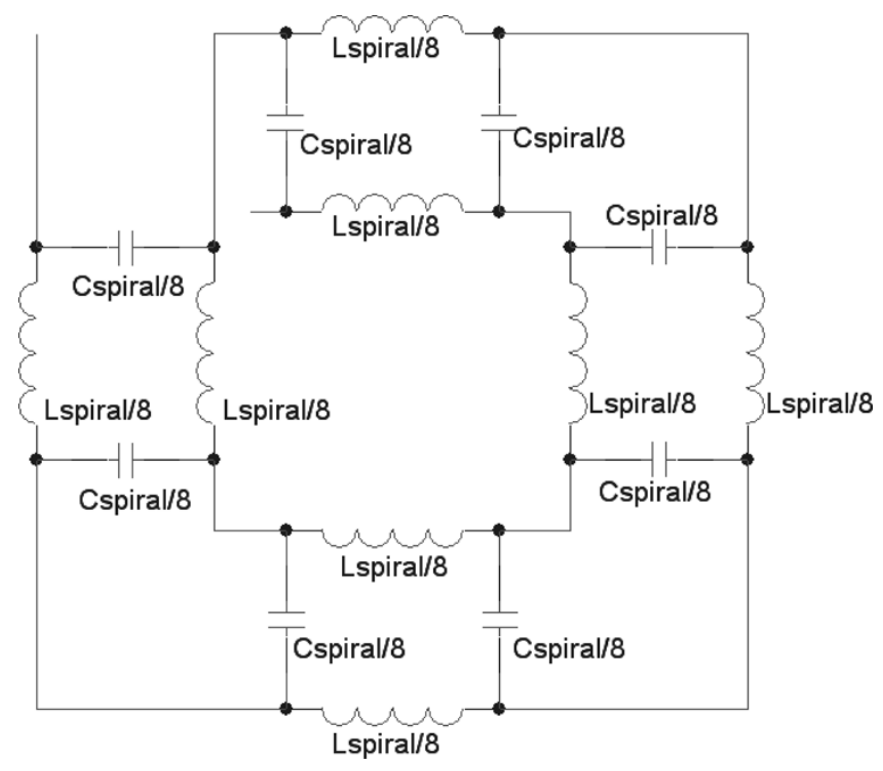

Fig. 10. Higher order spiral-loop equivalent-circuit model.

3) Resonance: Once the distributed capacitance and inductance of the spiral loop are known from the above methods, the resonance frequency of the EC can be estimated from

$$
F_{\text {res }}=\frac{1}{2 \pi \times \sqrt{L_{\text {spiral }} C_{\text {spiral }}}} .
$$

The estimate of resonance frequency from (6) is generally low. This estimation is partially due to estimation error of capacitance and inductance, but is also caused by the distributed nature of capacitance and inductance being poorly modeled by lumped elements. Equation (6) treats the capacitance and inductance as lumped values (as in Fig. 6), whereas they are actually distributed. For our case, (6) predicts a resonance frequency of $106 \mathrm{MHz}$, a dramatic underestimate of the realized values. At significantly increased complexity, the lumped values $C_{\text {spiral }}$ and $L_{\text {spiral }}$ may be distributed in a geometry more closely representing the actual spiral geometry (Fig. 10). This more accurately represents the interaction between the distributed capacitance and inductance, predicting a much higher resonance frequency of $183 \mathrm{MHz}$, but it is still too low relative to the actual spiral resonance and is not satisfactory for design purposes.

To more accurately model the medium, a finite-element solver such as the commercially available HFSS is helpful. Perfect electrically conducting (PEC) walls and perfectly magnetically conducting (PMC) boundary conditions around a single element unit can be employed to enforce symmetry conditions, which would exist in an infinite $Y Z$-plane of ECs under plane-wave illumination at normal incidence, as shown in Fig. 11.

The resonant frequency derived by this numerical simulation method will be quite accurate, although experimental errors will remain. For example, small air gaps between layers stacked in the $Y$-dimension may slightly decrease capacitance for physically realized materials and increase resonance frequency by $10 \%$ or $20 \%$ if not accounted for in the numerical simulations.

4) Effective Medium: In order to form an effective medium as represented by this model, a planar array of the unit cell of

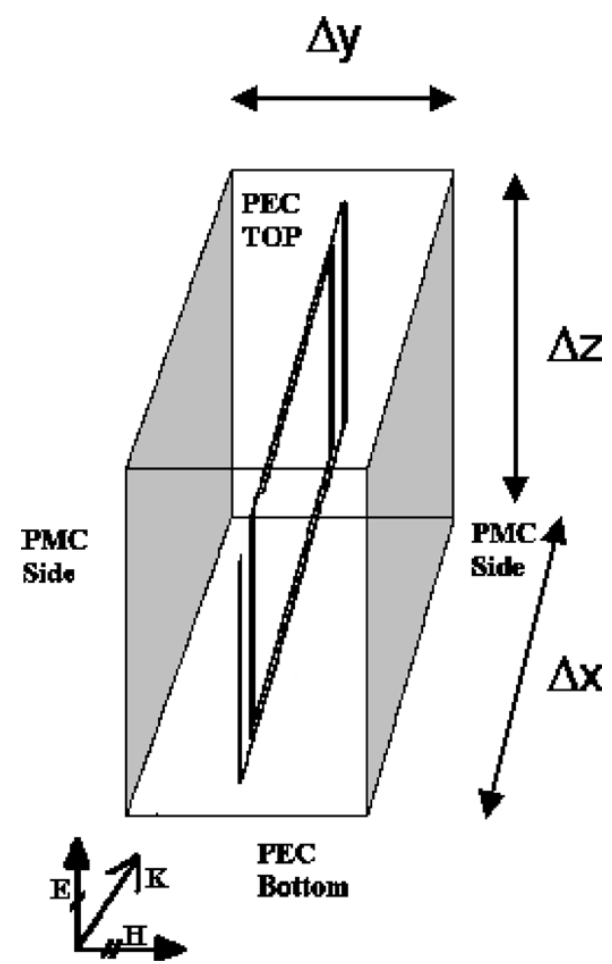

Fig. 11. Finite-element method (FEM) boundary conditions to test resonance frequency.

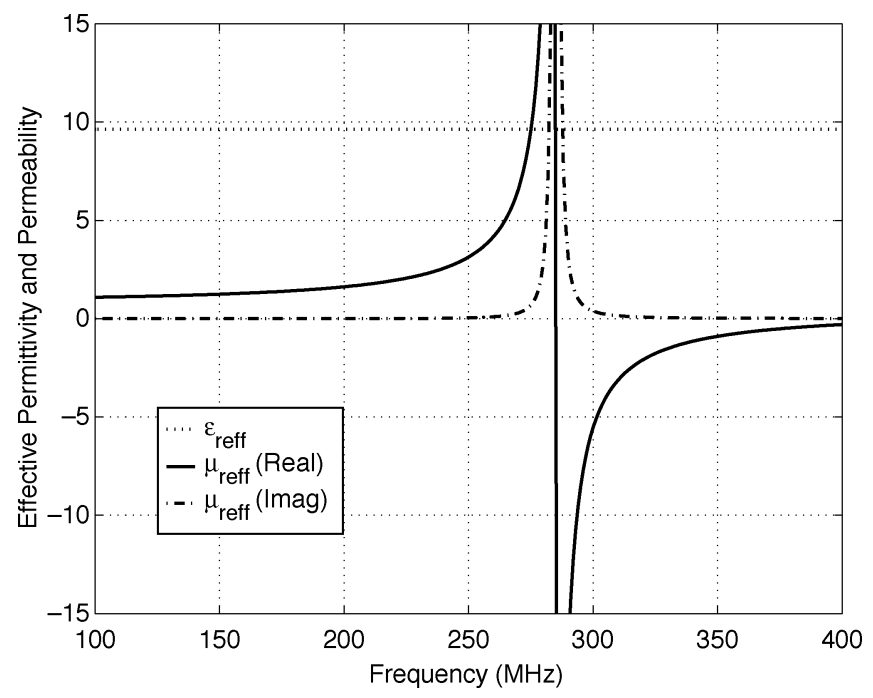

Fig. 12. Relative permittivity and permeability of metamaterial. At $250 \mathrm{MHz}$, $\epsilon_{r(\text { meta })}=9.8, \mu_{r(\text { meta })}=3.1$, and $\tan \delta_{m} 0.014$.

Fig. 3 is printed onto an $X Z$ planar surface. These infinite $X Z$ grid planes may be stacked infinitely in the $Y$-dimension to form a 3-D infinite medium.

Analytic formulations for the effective bulk permittivity and permeability of such EC metamaterials exist that correlate to the geometry of Fig. 7 [1]. The permeability and permittivity of such a medium are given in Fig. 12.

5) Permeability: Equation (7) gives the form of the anisotropic magnetic permeability. The effective-medium design provides permeability enhancement only along the solenoidal axis, which is parallel to the $Y$-axis. Any incident magnetic field of $X$ - or $Z$-orientation will not couple to the 
inductive loops of Fig. 4 and the permeability $\mu_{r}$ experienced by these components will be that of free space.

For a $Y$-oriented time-harmonic magnetic field, incident magnetic energy induces currents in the circuit loop coupling energy into the resonators and changing the relative permeability of the medium. The current loop induced generates its own magnetic field, storing magnetic energy and thereby changing the magnetic susceptibility

$$
\begin{aligned}
\overline{\bar{\mu}} & =\left[\begin{array}{ccc}
\mu_{0} & 0 & 0 \\
0 & \mu_{\mathrm{eff}} & 0 \\
0 & 0 & \mu_{0}
\end{array}\right] \\
\mu_{\mathrm{eff}} & =\mu_{0}\left(1-\kappa^{2} \frac{1}{1-\frac{\omega_{p}^{2}}{\omega^{2}}-\frac{j}{Q}}\right) .
\end{aligned}
$$

Effective permeability $\left(\mu_{\mathrm{eff}}\right)$ is given by (8) and is a function of the resonant frequency of the spiral inductors $\left(\omega_{p}=\right.$ $\left.2 \pi \times F_{\text {res }}\right)$, the frequency of the incident field $(\omega=2 \pi \times F)$, the resonator quality factor $Q$, and the coupling coefficient of $Y$-directed magnetic energy $\kappa$. Fig. 12 shows a typical response of $\mu_{\text {eff }}$ to frequency variation. Operating values of $\mu_{r}=1-5$ can be achieved with moderately low-loss performance.

The coupling coefficient $\kappa$ and most other metamaterial properties are a function of the circuit geometry shown in Fig. 3 as follows:

$$
\begin{aligned}
\kappa^{2} & =\frac{l_{x} l_{z}}{\Delta x \Delta z}<1 \\
Q & =\frac{2 l_{x} l_{z} w}{\Delta y\left(l_{x}+l_{z}\right) \delta} .
\end{aligned}
$$

The resonator $Q$ in (10) is a function of the conductor conductivity $\sigma$. Care should be taken to observe the condition that conductor thickness $\tau>2 \delta$, where $\delta=\sqrt{2 / \omega \mu_{0} \sigma}$ is the metallization skin depth at the frequency of operation.

6) Permittivity: Consider an $X$-directed electric field. Along the majority of the $X$-dimension, the electric field is shorted by the metallization of EC loop parallel to the incident $E$-field. In the gap region between the unit cells, the $Z$-directed metallizations form inter-cell capacitors for the incident $X$-directed electric field. This capacitance is what stores electrical energy and provides for the $X$-directed permittivity of (11). The same phenomenon is observed in the $Z$-dimension, but electric-field components oriented along the $Y$-dimension will experience the permittivity of the host dielectric only and $\epsilon_{r}=\epsilon_{r \text { diel }}$. The corresponding permittivity tensor is given by (12) as follows:

$$
\begin{aligned}
\epsilon_{\text {eff }} & =\epsilon_{\text {diel }}\left[1+\frac{\Delta z l_{x}}{\Delta x \Delta y} \frac{\left.K\left(\sqrt{1-g^{2}}\right)\right)}{K(g)}\right] \\
\overline{\bar{\epsilon}} & =\left[\begin{array}{ccc}
\epsilon_{\text {eff }} & 0 & 0 \\
0 & \epsilon_{\text {diel }} & 0 \\
0 & 0 & \epsilon_{\text {eff }}
\end{array}\right] .
\end{aligned}
$$

Unlike the permeability, the effective permittivity of the medium is not frequency dependent in the microwave region.

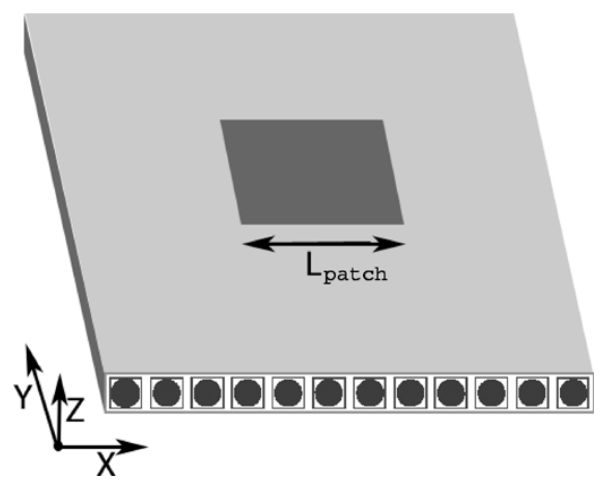

Fig. 13. Patch antenna over magnetic metamaterial substrate. Length $L_{\text {patch }}$ is the resonant length and indicates orientation of radiating current.

The permittivity in (11) is a function of the inter-cell capacitance, which is calculated by means of the same elliptical integral that was used to find the spiral capacitance (4). It should be noted that this simplification ignore the effects of metallization thickness and inter-cell capacitance due to the inner loops of the spirals and as such will underestimate the true capacitance and, hence, underestimate $\epsilon_{\text {eff }}$. Here, again, we seek physical intuition rather than computational precision.

This analysis assumes a medium where the circuits are embedded in a "simple" dielectric with $\mu_{r}=1$.

As this analysis has indicated, the metamaterial substrate will exhibit a highly anisotropic behavior. Permeability enhancement will be achieved for $Y$-directed magnetic fields only. Permittivity enhancement will occur only for $X$ - or $Z$-directed electric fields. This combination of orientation-dependent permeability and permittivity is exactly the orientations needed to support the modes of a microstrip patch antenna.

Fig. 13 demonstrates the proper orientation for a patch antenna operating in the regular mode to experience both $\mu_{\text {eff }}$ and $\epsilon_{\text {eff }}$. In the area under the patch, the image reflections from the metallic antenna and ground plane appear to form an infinite medium in the $Z$-dimension, allowing the effective medium analysis above to approximately apply despite the finite geometries.

\section{Metamaterial Design AND FABRicAtion}

The metamaterial substrate was designed with a cell size of $\Delta x=\Delta z=2 \mathrm{~cm}, \Delta y=3.028 \mathrm{~mm}(120 \mathrm{mil})$. The substrate was fabricated on 120-mil-thick Rogers RO-4003 dielectric. The spiral resonators were etched from $0.5-\mathrm{oz}$ thick copper $(0.017 \mathrm{~mm})$ with a linewidth $(w)$ and spacing $(s)$ of $0.127 \mathrm{~mm}$ (5 mil). For our design, $l x=l z=16 \mathrm{~mm}, w=s=0.127 \mathrm{~mm}$ and $L_{\mathrm{SG}}=6.24 \mathrm{~mm}$. For a Rogers RO-4003 dielectric host medium, $\Delta y=3.028 \mathrm{~mm}$ thick, $\epsilon_{r_{\text {diel }}}=3.38$ and $C_{\text {spiral }}=$ $5.3 \mathrm{pF}, N=2, S \approx 2.56 \times 10^{-4} \mathrm{~m}^{2}$ and $L_{\text {spiral }}=.425 \mu \mathrm{H}$. The resonance frequency predicted by (6) is, therefore, $106 \mathrm{MHz}$, but for the reasons previously described, this is an unreliable number and numerical simulation is used to identify the actual resonance frequency, which is $250 \mathrm{MHz}$.

To investigate the potential range of metamaterial operations, numeric simulations have been performed of the basic metamaterial unit cell (Fig. 3) under various conditions. With industry standard copper etching standards of 5-mil trace/5-mil 


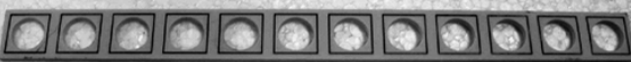

Fig. 14. $2 \times 24 \mathrm{~cm}$ strip of RO-4003 with 12 resonant loop unit cells.

spacing, on low-loss dielectrics it was possible to achieve metamaterial resonances at frequencies as low as $10 \mathrm{MHz}$ and as high $10 \mathrm{GHz}$. Physical measurements of metamaterial resonators with $2.5-\mathrm{GHz}$ resonances were also performed [4]. It seems likely that these materials can prove useful over a wide range of frequencies of operation.

To reduce substrate mass, 33/64-in-diameter air holes were drilled along the $y$-axis into the center of each spiral resonator cell. The final substrate mass is reduced by a factor of approximately $1 / 3$, which is significant for a 2 -cm-thick substrate. Fig. 14 shows a typical strip from which the final substrate was assembled. To determine the effects of these air holes, a numerical analysis of Fig. 11 with and without air holes was performed in HFSS. It was found that the inclusion of these air holes reduced the effective permittivity of the medium along the $X$ - and $Z$-axis by only $5 \%$. These drill holes do not strongly influence the substrate EM properties because the majority of electric-field energy storage occurs in the "gap" region between cells and the magnetic-field storage is unaffected by air gaps.

The final substrate was formed by stacking $X Z$-planar strips in the $Y$-dimension to form the final substrate in the geometry of Fig. 13. The final substrate was 24 cells in the $X$-dimension by 75 cells in the $Y$-dimension and one cell in the $Z$-dimension. The resulting total substrate was $24 \mathrm{~cm} \times 24 \mathrm{~cm} \times 2 \mathrm{~cm}$ and had a weight of approximately $3.5 \mathrm{lb}$.

\section{RESUlts}

\section{A. Metamaterial Performance}

With an assembled substrate, the transmission through the medium was measured by electrically small linear probes and a network analyzer, as shown in Fig. 15. Theory predicts such a medium to exhibit a nonpropagating condition at resonance. This was observed as a strong drop in measured transmission through the substrate medium at $285 \mathrm{MHz}$. By this means, the EC metamaterial resonance frequency $\left(F_{\text {res }}\right)$ was identified to be $285 \mathrm{MHz}$. The substrate was originally designed by numerical simulation for a resonance frequency of $250 \mathrm{MHz}$, and this discrepancy (14\%) can be explained by considering the effect of changes in spiral capacitance due to unmeasurable, but unavoidable air-gaps caused by imperfect stacking of substrate layers (Fig. 9). To investigate the effects of such air gaps, numerical simulation was performed to consider the effects of a $0.05-\mathrm{mm}$ air gap on our design. Simulations indicate that such a condition would increase the metamaterial resonance frequency of our design by $15 \%$, and this seems to validate the hypothesis that these air gaps are the majority cause of deviation in resonant frequency between measurement and the numerical simulation of the original design.

$\epsilon_{r \text { eff }}$ and $\mu_{r \text { eff }}$ for this design are predicted in Fig. 12. Equations (11) and (8) predict that, at $250 \mathrm{MHz}, \epsilon_{r \text { eff }}=9.8, \mu_{r \text { eff }}=$ 3.1 , and $\tan \delta_{m}=0.014$.

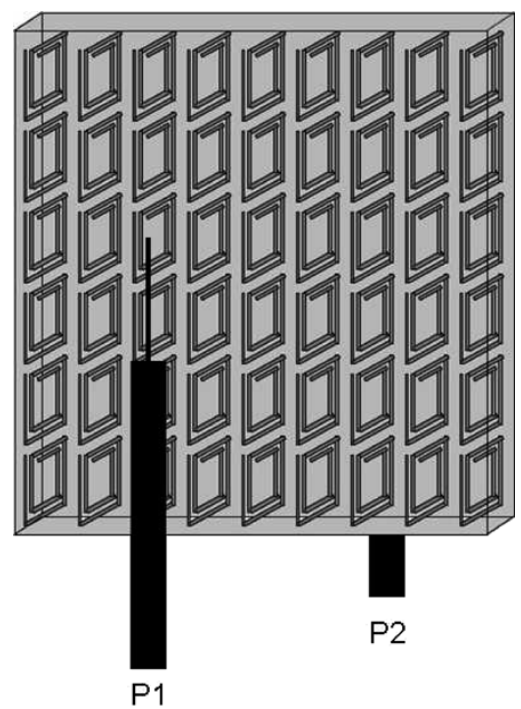

Fig. 15. Free-space transmissivity/reflectivity test of metamaterial substrate to identify resonance frequency.

To better characterize the medium, an independent measurement of $\epsilon_{r \text { eff }}$ is also desirable. The analysis of the effective medium assumes an infinite periodic array of EC elements, but obviously this is not the actual case for a metamaterial substrate. However, this approximation is valid in the area under the antenna patch due to the images from the patch and ground metallizations. In accordance with classical image theory, the region between two parallel metallic conductors such as the patch and its ground plane can be analyzed as equivalent to an infinite array whose period is the distance between the metallic plates. Therefore, our infinite medium analysis will approximately apply to our application case of a patch over a ground plane. To measure the achieved permeability, a test structure similar to the patch antenna geometry is required. The frequency independent form for permittivity given in (11) indicates that a low-frequency measurement of a parallel-plate metamaterial capacitor would give a reasonable estimate of the high-frequency permittivity in the patch-antenna design if the host dielectric itself is nondispersive.

To accomplish this, a large parallel-plate capacitor was constructed on the metamaterial substrate and measured at $2 \mathrm{MHz}$ with an Agilent E4991A impedance analyzer. The host dielectric itself (Rogers-RO4003) possesses a permittivity of only $\epsilon_{\text {diel }}=3.38$ at $2.5 \mathrm{GHz}$, whereas the parallel-plate capacitor fabricated from the EC metamaterial substrate exhibited an effective permittivity of $\epsilon_{r \text { eff }}=13.13$. This clearly shows permittivity enhancement at a level even slightly higher than was predicted. This deviation from theory (11) may be due to fringing fields coupling into the under hanging and adjacent loops, or variances in the distances between the spiral edge and the ground-plane or patch metallization. This distance, which is on the order of $2 \mathrm{~mm}$, may vary in experimentation by as much as $1 \mathrm{~mm}$.

\section{B. Antenna Performance}

Consideration of the field orientations of a patch antenna is beneficial at this time. Fig. 16 shows the basic geometry of our 


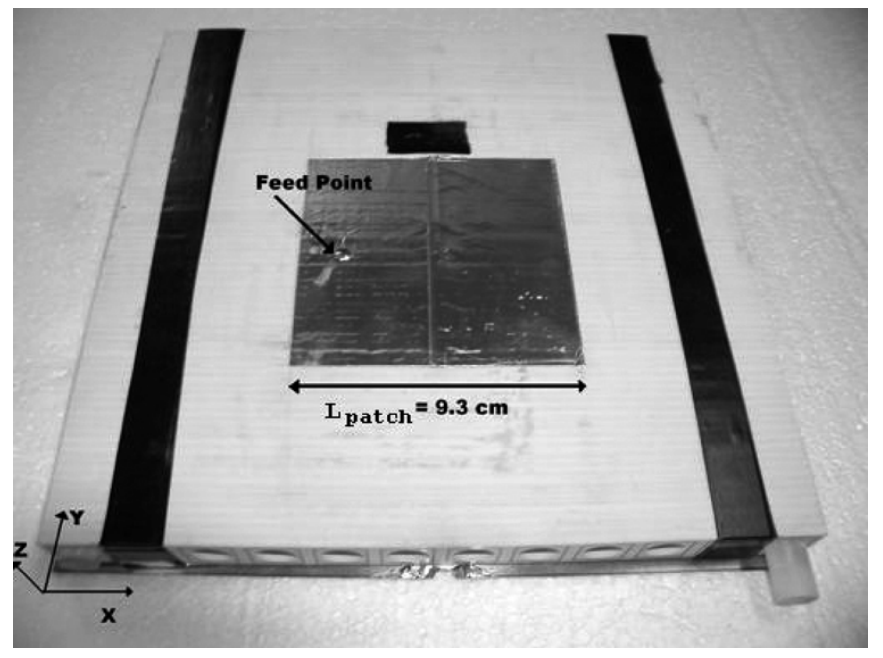

Fig. 16. Miniaturized microstrip patch antenna on a magnetic metamaterial substrate operating at $250 \mathrm{MHz}$. The resonant length $L_{\text {patch }}=0.077 \lambda=$ $9.3 \mathrm{~cm}$.

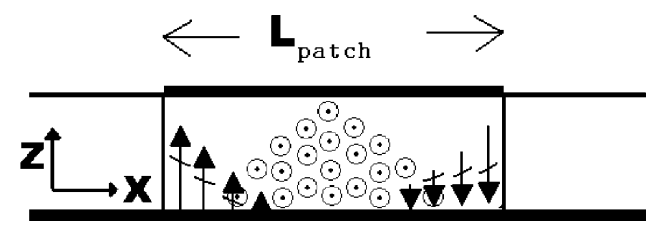

Fig. 17. Electric- and magnetic-field configurations in region beneath microstrip patch antenna. The electric field is aligned along the $Z$-axis and the magnetic field is aligned along the $Y$-axis normal to the page as shown.

patch antenna, where the resonant length is indicated by dimension $L$. A cross section taken along the $X Z$-plane in the middle of the patch antenna shows the field orientations of the dominant $T M Z_{010}$ in the substrate in Fig. 17. The electric fields are oriented along the vertical $Z$-axis and the magnetic fields are oriented along the $Y$-axis. These orientations correspond to the directions of electric permittivity and magnetic permeability enhancement, as established previously. If the patch is oriented, as shown in Fig. 16, the magnetic properties of this metamaterial will be observed, but a patch rotated one-quarter turn over the substrate with the resonant length $L$ along the $Y$-axis will experience only dielectric enhancement.

A probe-fed microstrip patch antenna resonant at $250 \mathrm{MHz}$ was built on the metamaterial substrate. To resonate at $250 \mathrm{MHz}$, the patch dimensions were found to be $9.3 \times 9.3 \mathrm{~cm}$. The final substrate plus patch antenna assembly is shown in Fig. 16 while the measured antenna gain pattern is shown in Figs. 18 and 19 shows the antenna return loss.

As mentioned earlier, this metamaterial substrate can be used to design patch elements with different miniaturization factors since $\mu_{\mathrm{eff}}$ is a function of frequency. For a material employing simple dielectrics, the relationship between operating frequency and physical geometry is fixed to a single miniaturization factor. By decreasing the physical dimensions by a factor of 2, the operating frequency increases by a factor of 2 and the miniaturization ratio remains constant. In contrast, for this metamaterial,

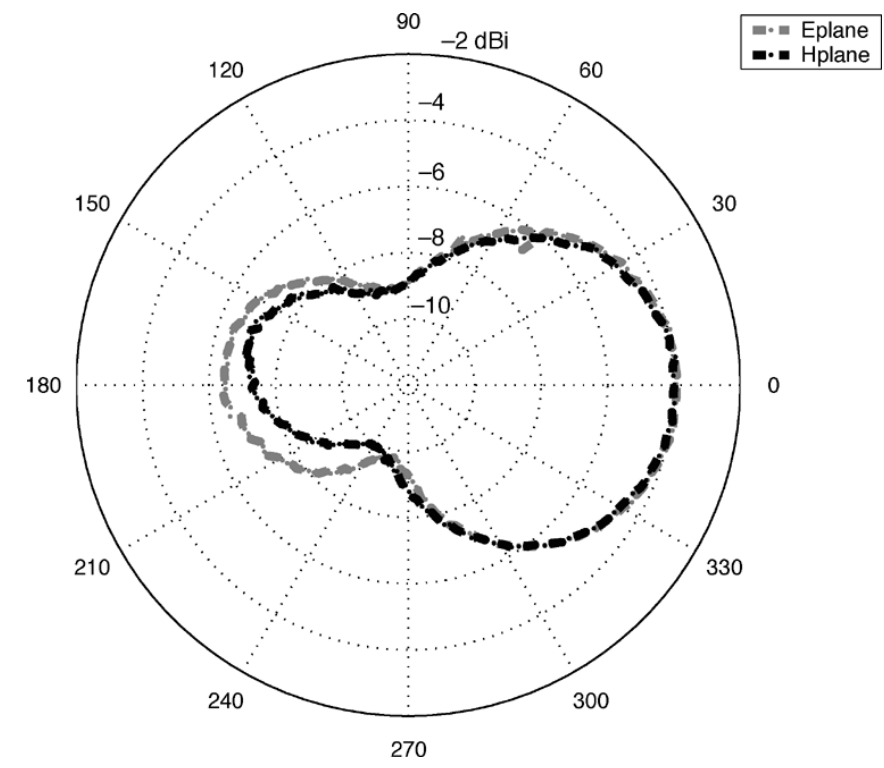

Fig. 18. Measured antenna gain pattern for patch antenna over magnetic metamaterial substrate at $250 \mathrm{MHz}$.

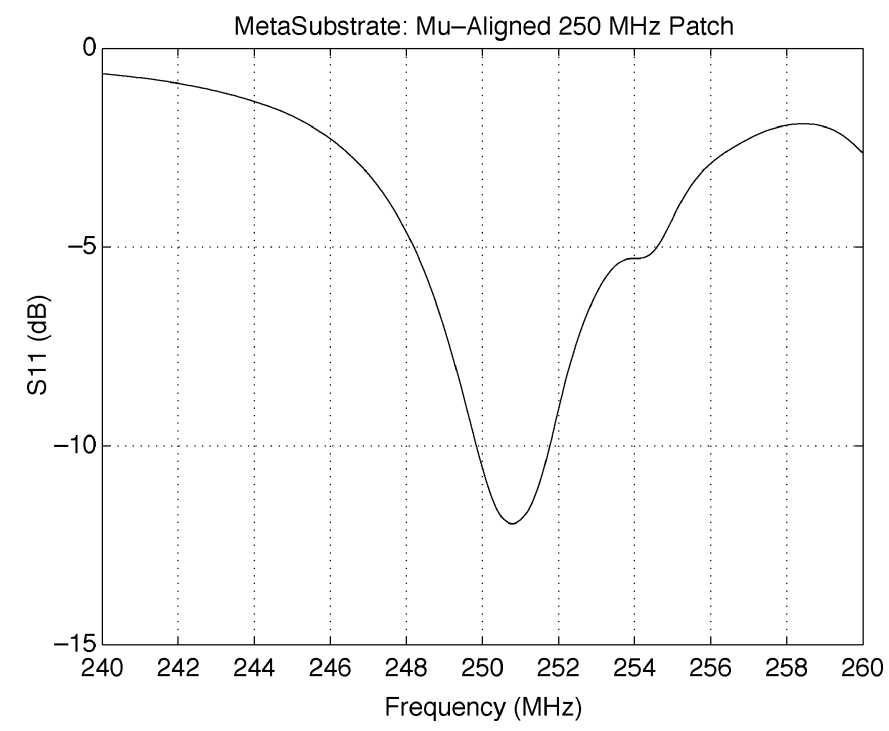

Fig. 19. Return loss at $250 \mathrm{MHz}$ for probe-fed patch antenna over magnetic metamaterial substrate.

it is possible for a $50 \%$ decrease in physical geometries to necessitate only a $20 \%$ increase in operating frequency. Therefore, with this metamaterial, the miniaturization factor is a function of operating frequency.

The antenna performance parameters, such as the miniaturization and efficiency factors of several patch antennas over the metamaterial substrate operating at different frequencies, are given in Tables I and II. A patch antenna in free space will resonate with a length of approximately $\lambda_{0} / 2$ and, for our purposes, the miniaturization factor is defined as the fraction of this size for which the patch resonates. For example, a miniaturization factor of 5 would indicate that the resonant length of the patch is $\lambda_{0} / 10$. 
TABLE I

ANTENNA PARAMETERS

\begin{tabular}{|c|c|}
\hline \multicolumn{2}{|c|}{$\begin{array}{c}0.077 \lambda \text { Patch Antenna at } 250 \mathrm{MHz} \\
\text { over Magnetic Metamaterial Substrate }\end{array}$} \\
\hline Return Loss & $-11.9 \mathrm{~dB}$ \\
-10dB RL BW & $0.83 \%$ \\
Gain & $-3.9 \mathrm{dBi}$ \\
Directivity & $3.4 \mathrm{dBi}$ \\
Cross-Pol Ratio & $-12 \mathrm{~dB}$ \\
Efficiency & $19.8 \%$ \\
Miniaturization Factor & 6.45 \\
\hline
\end{tabular}

TABLE II

MiNIATURIZATION AND EFFICIENCY

\begin{tabular}{|c|c|c|c|c|}
\hline Description & Frequency & $\begin{array}{c}\text { Resonant } \\
\text { Length }\end{array}$ & $\begin{array}{c}\text { Miniaturization } \\
\text { Factor }\end{array}$ & Efficiency \\
\hline Aligned & $215 \mathrm{MHZ}$ & $150 \mathrm{~mm}$ & 4.65 & $31.92 \%$ \\
Aligned & $240 \mathrm{MHZ}$ & $125 \mathrm{~mm}$ & 5.00 & $27.35 \%$ \\
Aligned & $250 \mathrm{MHZ}$ & $93 \mathrm{~mm}$ & 6.45 & $19.80 \%$ \\
Anti-Aligned & $250 \mathrm{MHZ}$ & $195 \mathrm{~mm}$ & 3.08 & $10.84 \%$ \\
\hline
\end{tabular}

Our procedure for measuring efficiency was by application of the relationship between gain and directivity. The maximum value of the gain is related to the maximum value of the directivity by [19]

$$
G_{0}=e D_{0} .
$$

The maximum gain $\left(G_{0}\right)$ of the antenna under test was measured experimentally by comparison to an antenna of known gain. To determine directivity, pattern measurements were taken along the primary $E$ - and $H$-plane cuts. Since this is a low-directivity antenna, these measurements were sufficient to approximate the total antenna pattern, and directivity was calculated from the classic formula [19]

$$
D_{0}=4 \pi \frac{U_{0}}{\int_{0}^{2 \pi} \int_{0}^{\pi} U(\theta, \phi) \sin (\theta) d \theta d \phi} .
$$

$U(\theta, \phi)$ is the directional radiation intensity there and $U_{0}$ is the radiation intensity in the direction of maximum radiation. Cross-polarization was also measured, but found to be low enough for these antennas that polarization efficiency was negligible to within the accuracy of the other estimates in this calculation.

To provide comparison, the antenna probe feed position and geometry were also adjusted to provide a resonant dimension along the $Y$-axis rather than the used previously $X$-axis (see Fig. 13). In this orientation, there should be no effective permeability experienced by the antenna, but permittivity should remain enhanced. That is $\mu_{r}=1$, but $\epsilon_{r \text { eff }}$ is given by (11).

As shown in Table II, the antialigned $250-\mathrm{MHz}$ case exhibits a miniaturization of just greater than three, which is predicted by the theoretical and measured permittivity enhancement alone

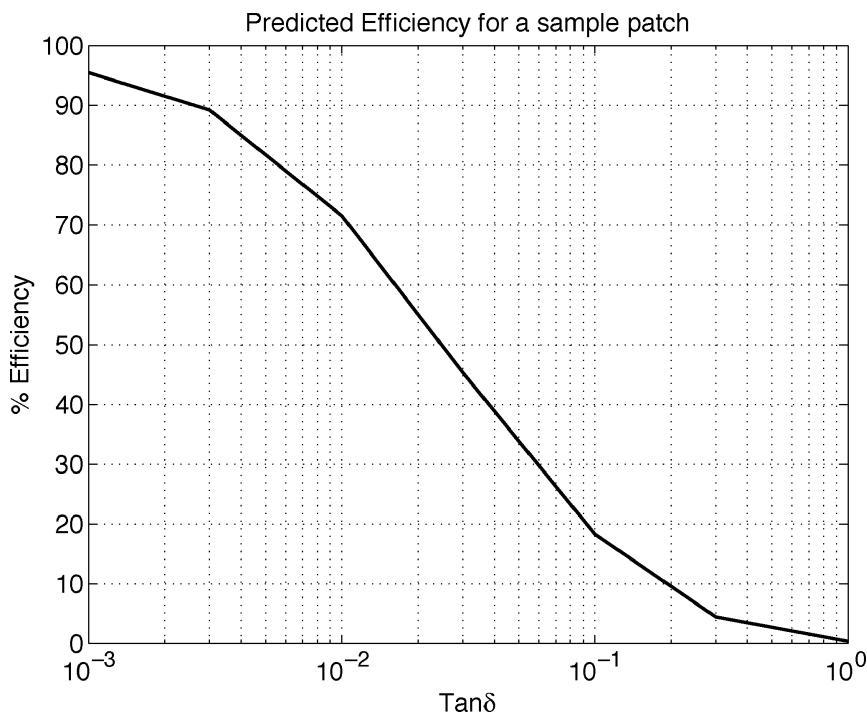

Fig. 20. Radiation efficiency of a patch at $250 \mathrm{MHz}$ verses substrate material loss tangent on modeled metamaterial substrate.

with no permeability enhancement. In this patch orientation, the magnetic enhancement of the material does not provide for miniaturization and, as a result, the patch is comparable to the size of the ground plane. This nearly parallel-plate capacitor structure suffers from somewhat stronger edge effects and, therefore, should exhibit lower efficiency. That the nonmagnetic orientation of operation yields significant losses indicates that the losses observed in the permeability-enhanced orientation are not entirely caused by the magnetic loss tangent itself. The ohmic losses of the patch, ground plane, and dielectric loss tangent of the substrate are also contributing to the high losses.

The orientation, frequency dependence, and observed miniaturization factor values reinforces validation of our EC spiral loop resonator model.

Fig. 19 shows the insertion loss of the $9.3 \times 9.3 \mathrm{~cm}$ patch antenna around $250 \mathrm{MHz}$. This figure indicates a more difficult input matching condition than the bulk material properties $\epsilon_{\mathrm{eff}}$, $\mu_{\text {eff }}$ would imply. This is due to the material bulk properties not being as well representative of the very near-field properties around the probe feed. The probe feed does not induce a simple field geometry to interact ideally with the metamaterial anisotropies the way that the patch itself does. Even so, this is an acceptable feed condition to demonstrate the properties of metamaterial substrates.

The observed efficiencies in this experiment are lower than would be predicted by (8). This is at least partially due to the overly simplifying assumption of (10). The $0.5-\mathrm{oz}$ copper $(\sigma=5.8 E 7 \mathrm{~S} / \mathrm{m})$ used as our metallization has a skin depth of $10.5 \mu \mathrm{m}$ at $250 \mathrm{MHz}$. Since $0.5-\mathrm{oz}$ copper is only $17 \mu \mathrm{m}$ in thickness, (10) predicts a higher performance than is realizable with this metallization. Numerical simulation indicated that a thicker metallization $70 \mu \mathrm{m}(2 \mathrm{oz})$ will improve the magnetic loss tangent by a factor of almost 2 , and this should improve antenna efficiency levels. An additional source of loss is the dielectric loss tangent of the host dielectric material. Numerical simulation indicates that decreasing the host material dielectric lost tangent will significantly improve the efficiency of the 
effective medium, but not as strongly as improvements to the metallization would.

To estimate the impact of changes in material loss tangent on antenna efficiency, an anisotropic substrate was modeled according to (7) and (12). With a copper patch and ground plane $35-\mu \mathrm{m}$ thick on a 2 -cm-thick substrate $\left(\epsilon_{r \text { diel }}=3.38, \epsilon_{\text {reff }}=\right.$ 9.8, $\mu_{\text {reff }}=3.1$, and $\tan \delta=\tan \delta_{e}=\tan \delta_{m}$ ), numerical analysis of a $250-\mathrm{MHz}$ patch was performed using HFSS software (Fig. 20).

\section{CONCLUSION}

A naturally nonmagnetic dielectric was imbued with magnetic properties and permeability by means of embedded resonant circuits. This metamaterial substrate possesses enhanced positive values of permeability with reasonable loss levels. A microstrip patch antenna was developed and tested to demonstrate the potential application of these EC magnetic metamaterial substrates. Miniaturization factors on the order of 4-7 and moderate efficiencies of $20 \%-30 \%$ were observed, validating that various miniaturization factors may be selected in a miniaturization/efficiency optimization using a single material. Higher efficiencies should be achievable with judicious metallization choice. This experiment indicates the validity of the EC resonator metamaterial analytical model. It is predicted that a wide range of applications will benefit from moderate or lowloss magnetic metamaterials in the microwave region and that some applications will benefit from tunable miniaturization.

EC metamaterials like those described here have been fabricated for applications of up to $2.5 \mathrm{GHz}$. Simulations indicate that with commercially available low-dielectric materials and standard 5-mil commercially available processing technology, magnetic metamaterials produced by this technique can be designed with an operating frequency of up to $10 \mathrm{GHz}$ with permeability in the $\mu_{r}=1-5$ range for moderately low-loss applications.

\section{REFERENCES}

[1] K. Sarabandi and H. Mosallaei, "Electro-ferromagnetic tunable permeability, band-gap, and bi-anisotropic meta-materials utilizing embeddedcircuits," in IEEE AP-S Int. Symp., Columbus, OH, Jun. 22-27, 2003. [Digital Archive].

[2] - "Design and characterization of a meta-material with both $e-m$ parameters realized utilizing embedded-circuit artificial molecules," IEEE Trans. Antennas Propag., Dec. 2004, submitted for publication.

[3] H. Mosallaei and K. Sarabandi, "Magneto-dielectrics in electromagnetics: Concept and applications," IEEE Trans. Antennas Propag., vol. 52, no. 6, pp. 1558-1567, Jun. 2004.

[4] K. Buell, H. Mosallaei, and K. Sarabandi, "Measurement of meta-materials utilizing resonant embedded-circuit for artificial permeability by frequency extended perturbation method," in IEEE AP-S Int. Symp., Columbus, OH, Jun. 22-27, 2003. [Digital Archive].

[5] M. M. Saadoun and N. Engheta, Theoretical Study of Electromagnetic Properties of Nonlocal 'Omega' Media, ser. PIER Monograph, A. Priou, Ed., 1994, vol. 9, Bi-anisotropic Bi-isotropic Media Applicat., ch. 15, pp. 351-397.

[6] J. B. Pendry, A. J. Holden, D. J. Robbins, and W. J. Stewart, "Magnetism from conductors and enhanced nonlinear phenomena," IEEE Trans. Microw. Theory Tech., vol. 47, no. 11, pp. 2075-2081, Nov. 1999.

[7] D. M. Pozar, Microwave Engineering, 2nd ed. New York: Wiley, 1997.

[8] W. D. Callister, Jr., Materials Science And Engineering: An Introduction. New York: Wiley, 2003, ch. 21.
[9] V. G. Veselago, "The electrodynamics of substances with simultaneously negative values of $\epsilon$ and $\mu$, Sov. Phys.-Usp., vol. 10, pp. 509-514, Jan.-Feb. 1968.

[10] N. Engheta, "Electromagnetics of complex media and metamaterials," in Mathematical Methods in Electromagnetic Theory Int. Conf., vol. 1, Sep. 10-13, 2002, pp. 175-180.

[11] _ "Metamaterials with negative permittivity and permeability: Background, salient features, and new trends," in IEEE MTT-S Int Microwave Symp. Dig., vol. 1, Jun. 8-13, 2003, pp. 187-190.

[12] R. W. Ziolkowski, "Doubler negative metamaterial design, experiments, and applications," in IEEE AP-S Int. Symp., vol. 2, Jun. 16-21, 2002, pp. 396-399.

[13] - "Design, fabrication, and testing of double negative metamaterials," IEEE Trans. Antennas Propag., vol. 51, no. 7, pp. 1516-1529, Jul. 2003.

[14] K. Aydin, M. Mayindir, and E. Ozbay, "Microwave transmission through metamaterials in free space," in Quantum Electronics and Laser Science Conf. Tech. Dig., May 19-24, 2002, p. 12.

[15] N. C. Panoiu and R. M. Osgood, Jr., "Feasibility of fabricating metamaterials with negative refractive index in the visible spectrum," in IEEE Lasers Electro-Optics Tech. Dig., vol. 1, May, 19-24 2002, pp. 241-242.

[16] D. J. Kern and D. H. Werner, "The synthesis of metamaterial ferrites for RF applications using electromagnetic bandgap structures," in IEEE AP-S Int. Conf., vol. 1, Jun. 22-27, 2003, pp. 497-500.

[17] J. McVay, A. Hoorfar, and N. Engheta, "Radiation characteristics of microstrip dipole antennas over a high-impedance metamaterial surface made of Hilbert inclusions," in IEEE MTT-S Int. Microwave Symp. Dig., vol. 1, Jun. 8-13, 2003, pp. 587-590.

[18] A. Grbic and G. V. Eleftheriades, "A backward-wave antenna based on negative refractive index $L-C$ networks," in IEEE AP-S Int. Symp., vol. 4, Jun. 16-21, 2002, pp. 340-343.

[19] C. A. Balanis, Antenna Theory: Analysis and Design, 2nd ed. New York: Wiley, ch. 1997

Kevin Buell (S'89) received the B.Sc. degree in computer and systems engineering from Rensselaer Polytechnic University (RPI), Troy, NY, in 1999, and the M.S. and Ph.D. degrees in electrical engineering from The University of Michigan at Ann Arbor, in 2002 and 2005, respectively.

Mr. Buell was the recipient of the First Place Prize in the Student Paper Competition of the 2003 and 2005 IEEE Antennas and Propagation Society (IEEE AP-S) Symposium and was also the recipient of numerous scholarships and awards including the Intel Doctoral Fellowship, the Motorola Graduate Fellows Award, the Wynant J. Williams Award, and the Rensselaer Founders Award.

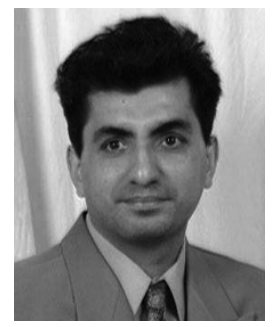

Hossein Mosallaei (SM'98) received the B.Sc. and M.Sc. degrees in electrical engineering from Shiraz University, Shiraz, Iran, in 1991 and 1994, respectively, and the Ph.D. degree in electrical engineering from the University of California at Los Angeles (UCLA), in 2001

He is currently an Assistant Professor of electrical and computer engineering with the College of Engineering, Northeastern University. From 2002 to 2005, he was on the faculty of the Electrical Engineering and Computer Science (EECS) Department, The University of Michigan at Ann Arbor, as an Assistant Research Scientist. He has authored or coauthored over 70 technical journal and conference papers. $\mathrm{He}$ is the holder of one U.S. patent with one pending patent. His research interests include applied electromagnetics and RF circuits with the focus on metamaterials and microscale/nanoscale devices and systems. He is listed in Who's Who in America.

Dr. Mosallaei is a full member of URSI and a member of the American Association for the Advancement of Science. He served as the vice-chair of the IEEE Michigan Trident Chapter [IEEE Antennas and Propagation Society (IEEE AP-S), IEEE Microwave Theory and Techniques Society (IEEE MTT-S), IEEE Electron Devices Society (IEEE ED-S)] in 2005. He has organized several special sessions in various IEEE conferences. He has also been a plenary session speaker in numerous national and international symposia. He was the corecipient of Student Prize Paper Awards presented at the IEEE AP-S (2000, 2001, 2003, and 2005), the 2001 URSI Young Scientist Award, and 2002 Raj Mittra Award. 


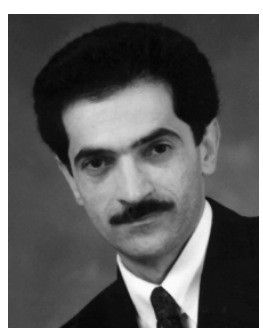

Kamal Sarabandi (S'87-M'90-SM'92-F'00) received the B.S. degree in electrical engineering from the Sharif University of Technology, Tehran, Iran, in 1980, and the M.S.E. and Ph.D. degrees from The University of Michigan at Ann Arbor, in 1986 and 1989 , respectively, both in electrical engineering.

He is currently Director of the Radiation Laboratory and a Professor with the Department of Electrical Engineering and Computer Science, The University of Michigan at Ann Arbor. His research interests include microwave and millimeter-wave radar remote sensing, metamaterials, EM-wave propagation, and antenna miniaturization. $\mathrm{He}$ possesses 20 years of experience with wave propagation in random media, communication channel modeling, microwave sensors, and radar systems and currently leads a large research group including two research scientists, ten $\mathrm{Ph} . \mathrm{D}$. students, and two M.S. students. Over the past ten years, he has graduated 21 $\mathrm{Ph} . D$. students. He has served as the Principal Investigator on many projects sponsored by the National Aeronautics and Space Administration (NASA), Jet Propulsion Laboratory (JPL), Army Research Office (ARO), Office of Naval Research (ONR), Army Research Laboratory (ARL), National Science Foundation (NSF), Defense Advanced Research Projects Agency (DARPA), and numerous industries. He has authored or coauthored many book chapters and over $125 \mathrm{pa}-$ pers in refereed journals on EM scattering, random media modeling, wave propagation, antennas, metamaterials, microwave measurement techniques, radar calibration, inverse-scattering problems, and microwave sensors. He has also had over 300 papers and invited presentations in many national and international conferences and symposia on similar subjects. He is listed in American Men and Women of Science, Who's Who in America, and Who's Who in Science and Engineering.

Dr. Sarabandi is a vice president of the IEEE Geoscience and Remote Sensing Society (GRSS), and a member of the IEEE Technical Activities Board Awards Committee. He is the associate editor of the IEEE TRANSACTIONS ON ANTENNAS AND PROPAGATION and the IEEE SENSORS JOURNAL. He is also a member of Commissions F and D of URSI and of The Electromagnetic Academy. He was the recipient of the Henry Russel Award presented by the Regent of The University of Michigan at Ann Arbor (the highest honor The University of Michigan at Ann Arbor bestows on a faculty member at the assistant or associate level), the 1999 GAAC Distinguished Lecturer Award presented by the German Federal Ministry for Education, Science, and Technology given to approximately ten individuals worldwide in all areas of engineering, science, medicine, and law, the 1996 Electrical Engineering and Computer Science Department Teaching Excellence Award, the 2004 College of Engineering Research Excellence Award, the 2005 IEEE Geoscience and Remote Sensing Distinguished Achievement Award, and the 2005 The University of Michigan Faculty Recognition Award. Over the past several years, joint papers presented by his students at a number of symposia [IEEE Antennas and Propagation Society (IEEE AP-S) Symposia (1995, 1997, 2000, 2001, 2003, 2005); the IEEE International Geoscience and Remote Sensing Symposium $(1999,2002)$; the IEEE Microwave Theory and Techniques Society (IEEE MTT-S) International Microwave Symposium (IMS) (2001), and URSI (2004, 2005)] have been the recipients of Student Prize Paper Awards. 